Rapport - Société canadienne d'histoire de l'Église catholique

\title{
Les débuts du Séminaire de Chicoutimi (1873-1888)
}

\section{Jean-Claude Drolet}

Volume 32, 1965

URI : https://id.erudit.org/iderudit/1007328ar

DOI : https://doi.org/10.7202/1007328ar

Aller au sommaire du numéro

Éditeur(s)

Les Éditions Historia Ecclesiæ Catholicæ Canadensis Inc.

ISSN

0318-6148 (imprimé)

1927-7075 (numérique)

Découvrir la revue

Citer cet article

Drolet, J.-C. (1965). Les débuts du Séminaire de Chicoutimi (1873-1888). Rapport

- Société canadienne d'histoire de l'Église catholique, 32, 29-35.

https://doi.org/10.7202/1007328ar

Tous droits réservés @ Les Éditions Historia Ecclesiæ Catholicæ Canadensis Inc., 1966
Ce document est protégé par la loi sur le droit d'auteur. L'utilisation des services d'Érudit (y compris la reproduction) est assujettie à sa politique d'utilisation que vous pouvez consulter en ligne.

https://apropos.erudit.org/fr/usagers/politique-dutilisation/ 


\section{Les débuts du Séminaire de Chicoutimi 1873-1888}

\section{INTRODUCTION}

L'œuvre religieuse et éducationnelle des évêques canadiens éclate maintenant à tous les yeux. Les archives, plus facilement exploitées par les historiens, témoignent éloquemment de cette œuvre. Mais ce qu'il faut faire connaître à la génération actuelle, c'est l'intelligence, la science, les vertus, la sage administration, l'énergie et le dévouement inlassables déployés par ces évêques dans la fondation des différentes régions du Canada. A l'exemple de Mgr François de MontmorencyLaval, premier évêque de la Nouvelle-France, les évêques canadiens ont été, pour la plupart, les grands bâtisseurs du Canada français et catholique. Parmi cette lignée impressionnante d'évêques bâtisseurs, missionnaires, colonisateurs, se dégage la belle et noble figure de Mgr Dominique Racine, fondateur de l'Eglise saguenéenne.

Il est évident que ce premier évêque du vaste diocèse de Chicoutimi a été un facteur puissant dans l'établissement et le développement du "Royaume du Saguenay ". L'œuvre de Mgr Racine est d'une portée immense, son action tenace et énergique s'est déployée dans plusieurs secteurs, mais son œuvre apostolique a porté surtout sur les domaines religieux et éducationnel. Parmi les nombreuses réalisations de a l'Apôtre du Saguenay » le Séminaire de Chicoutimi tient une place primordiale car il est l'œuvre de son cœur, son œuvre d'amour : " Mon Séminaire... Mon Séminaire, comme je l'aimais ${ }^{1}$ !" s'écrira le premier évêque de Chicoutimi à la veille de mourir.

\section{I. - SITUATION DU SAGUENAY VERS 1873}

Après avoir exercé son ministère à différents endroits de l'archidiocèse de Québec, l'abbé Dominique Racine est nommé quatrième curé de Chicoutimi en 1862. A cette date, Chicoutimi a vingt ans d'existence. C'est une paroisse qui sort à peine de la période de colonisation. Son village ressemble à un "rang", aux maisons espacées le long d'un chemin de terre, sans trottoirs, avec des granges et des hangars ici et là, une église de bois assez vaste mais pauvre vers le centre et des agglomérations aux deux extrémités, soit Rivière-du-Moulin et le Bassin, dans le voisinage des scieries.

Le Saguenay dont le curé Racine deviendra très tôt le vicaire forain, vicaire général et plus tard premier évêque, est un immense territoire peu peuplé, constitué de toute l'étendue du Royaume du Saguenay avec

1 L'Oiseau-Mouche, 29 septembre 1900, Vol. III, no 14. 
en plus le comté de Charlevoix. Le recensement de 1871 attribue à cette région une population de 17,473 habitants, population composée presque exclusivement de cultivateurs et de colons. Sur le plan religieux, le diocèse de Chicoutimi n'existera qu'en 1878. Tout ce territoire appartient alors à l'archidiocèse de Québec et comprend une douzaine de paroisses assez florissantes, d'autres sont en voie de formation. En somme c'est' un pays assez bien organisé sur les plans civil, religieux et éducationnel. Les villages de Chicoutimi et de Grande-Baie possèdent chacun une école modèle; ailleurs les enfants reçoivent leur instruction à

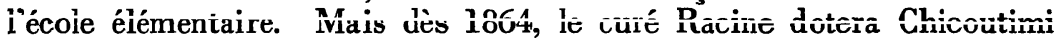
d'un couvent dirigé par les Sœurs du Bon-Pasteur, couvent qui apporte un progrès considérable, surtout au niveau de l'éducation, ceci par la formation d'institutrices, puisqu'il n'y avait pas d'école normale à cette époque. C'est un tableau assez encourageant pour une jeune région, mais est-ce suffisant pour le jeune et dynamique curé ? Dominique Racine prévoit une meilleure organisation religieuse pour ce territoire immense mais isolé du reste du pays. Dès son arrivée, il songe à doter le Saguenay d'une organisation plus adéquate qui lui permettra de se suffire à lui-même. Cet homme supérieur qui voit loin songe très tôt à la fondation d'un Séminaire à Chicoutimi.

\section{II. - NÉCESSITÉ D’UN SÉMINAIRE ET PREMIERES DÉMARCHES}

Convaincu de la nécessité d'une telle institution pour le Saguenay, le curé Dominique Racine communique ce projet à son ami l'abbé Thomas-Etienne Hamel, du Séminaire de Québec, dans une lettre du 19 octobre 1865 :

... J'ai un projet en tête - écrit-il - je te le communique et tu me diras ce que tu en penses. Je cherche les moyens de jeter les fondations d'un collège pour le Saguenay. Je pourrai bien me procurer une maison assez vaste avec un bon terrain; mais il faudrait terminer l'intérieur de la maison et construire des dépendances et il faudrait surtout se procurer de bons maîtres, mais où les prendre, où jeter les yeux. Je te dirai franchement que j'ai plus d'une fois pensé au Séminaire de Québec, j'étais même décidé à en parler à M. le Supérieur si je l'avais rencontré à Québec lors de mon voyage. Dis-moi donc franchement ce que tu penses de ce projet. Dis en donc un mot à $M$. le Supérieur; mais rappelle-t-oi que je ne voudrais pas que la chose fût ébruitée 2 .

Le pauvre curé de Chicoutimi a bien peu de ressources pour mettre à exécution son projet; la pauvreté d'ailleurs sera la compagne de toute sa vie. Puis bien souvent le Saguenay est à la misère, les récoltes sont quelquefois manquées, bien des familles souffrent et ont peine à se nourrir. Homme au tempérament de constructeur, il mûrit ce projet, en cause avec des amis. En 1866, il écrit de nouveau à l'abbé T.-E. Hamel :

Pour une idée Napoléon a fait la guerre d'Italie; et moi pour la réalisation d'une idée je fais la guerre à la patience d'un ami. Je suis

2 A.S.Q., Université 80, no 35. Lettre du Curé Dominique Racine à l'abbé T.-E. Hamel du Séminaire de Québec, datée du 19 octobre 1865. 
poursuivi par cette idée et plus j'y pense plus je trouve que s'il est une partie du Diocèse qui ait besoin d'un collège, c'est le Saguenay. Que je voudrais être riche, comme il s'en trouve sous la calotte du ciel, j'aurais bientôt mis hache en bois. Malheureusement ma bourse n'est pas aussi grande que mes désirs; et j'ai encore des créanciers qui crient après moi. Malgré cela je suis bien prêt à faire des sacrifices pour la réalisation de ce projet ${ }^{3}$.

L'entreprise du curé Racine est extrêmement difficile pour l'époque, il le sait bien, mais il a la foi et le zèle d'un apôtre - instrument de la Providence - c'est là sa seule force. Il plaide encore une fois pour un collège à Chicoutimi, en 1867, dans une autre lettre à l'abbé Hamel :

Comme toi et le Séminaire, je regrette la multiplicité des collèges et je voudrais pouvoir dire : on peut s'en passer. Mais le Saguenay se trouve dans une position exceptionnelle : nous sommes pour ainsi dire séparés du reste des mortels par nos hautes montagnes, nos voies de communication très difficiles en hiver et très coûteuses en été. Et puis tu sais que Nos Seigneura leq Evêques de la Province ont déjà parlé de former un Evêché dans le Saguenay. Or, tout cela joint aux faibles moyens de nos jeunes colons, me force à croire que nous ne pouvons appliquer au Saguenay la même règle que l'on voudrait appliquer ailleurs. N'importe, je ferai mon possible et si je ne réussis pas, ce sera la meilleure preuve que l'on doit s'en passer puisque Dieu ne me fournira pas les moyens de succès 4 .

Riche de sa seule confiance en Dieu et d'une volonté tenace, le curé Racine, déjà fort occupé par d'autres réalisations, remet à plus tard la fondation de son Séminaire. Des années 1867 à 1870 nos archives ne mentionnent plus de démarches à ce sujet. Les annales du Séminaire de Chicoutimi soulignent cependant, en 1870, son * désir de fonder à Chicoutimi une école où on enseignerait le français, l'anglais et la tenue des livres ${ }^{5}$. Puis dans une lettre du 10 avril 1872 adressée à Mgr l'archevêque E.-A. Taschereau, le curé de Chicoutimi expose à Sa Grâce la nécessité de commencer à Chicoutimi cette maison d'éducation qui deviendra plus tard un collège. Le 18 du même mois, Mgr l'Archevêque de Québec répond en ces termes :

... c'est très bien, et je donne de tout cœur ma bénédiction au futur Collège dont les humbles commencements permettent comme tout ce qui est humble, une grande prospérité et une longue durée... Je ferai tout en mon pouvoir pour l'aider et le favoriser... Courage !... Une auvre comme celle-là ne se fait pas en un jour ni sans des peines infinies. Vous serez tenté plus d'une fois de regretter de l'avoir entreprise, vous vous trouverez souvent en face des difficultés qui paraîtront insurmontables; mais ne craignez rien, car Dominus erit vobiscum et à la fin vous direz avec joie Bonum certamen certavi. Voilà donc la première pierre de l'édifice que je pose et que je bénis : vous allez dire peut-être qu'elle paraît lourde et hérissée d'angles menaçants. C'est néanmoins une pierre de la meilleure espèce ${ }^{b}$.

3 A.S.Q., Université 80, no 36, Lettre de D. Racine à l'abbé T.-E. Hamel, en date du 4 mars 1866.

4 A.S.Q., Université 105, 37, Lettre de M. D. Racine à M. T.-E. Hamel, du $1^{\text {er }}$ avril 1867 .

5 A.S.C., $A$ nn., vol. I, p. 1.

- A.S.C., $A n n$. , vol. I, p. 2-3. 
Encouragé par cette bénédiction de l'Archevêque de Québec, le curé Dominique Racine voit l'heure venue de jeter les bases de son Séminaire, de concrétiser enfin un rêve caressé depuis 1865, c'est-à-dire trois ans à peine après son arrivée comme curé de Chicoutimi.

\section{III. - RÉALISATION, FONDATION, DÉVELOPPEMENT}

Les commissaires d'école du village de Chicoutimi : Ovide Bossé, Méron Tremblay, Eucher Lemieux et Michel Caron offrent spontanément à leur curé la maison d'école du village; en conséquence, une résolution des commissaires est entrée dans le registre le 14 novembre 1872. L'acte de vente est passé le $l^{\text {er }}$ août 1873 et la maison d'école ainsi qu'une acre de terrain sont cédées à la corporation archiépiscopale pour le prix nominal d'un dollar. Dans le cours de l'hiver et de l'été de 1873, des réparations sont effectuées à cette maison au prix de $\$ 700$. Le curé Racine profite de la visite de confirmation de Mgr l'Archevêque de Québec pour lui faire bénir le nouveau collège le 28 juillet 1873. Le 15 août suivant, Mgr Taschereau érige par un décret canonique cette nouvelle maison en Séminaire de Chicoutimi et le place sous la protection de la sainte Famille, Jésus, Marie, Joseph. Le révérend Dominique Racine, vicaire général, en est le premier supérieur et M. Samuel Caron, curé de Saint-Louis de Métabetchouan, directeur ?

La première année scolaire débute le 15 septembre 1873, quarantecinq élèves sont présents en ce jour mais leur nombre s'élèvera à soixante et un dans le cours de l'année. Le 16, le curé J. Auclair, de Québec, célèbre une messe solennelle pour attirer la bénédiction de Dieu sur le nouveau Séminaire. Le nouveau supérieur prend son rôle à cœur et prêche lui-même la retraite des étudiants à la mi-novembre et fait subir l'examen du premier semestre, le $1^{\text {er }}$ février $1874{ }^{8}$. Vers le 18 juin, débute une tradition encore respectée de fêter le Supérieur du Séminaire. A la fin de l'année, il préside lui-même les examens.

L'installation dans cette maison d'école ne pouvant être que temporaire, Mgr Taschereau, dès le mois de mars 1874, lui demande de faire préparer le bois pour servir à la construction du futur Séminaire ${ }^{9}$. En cette même année Mgr l'Archevêque acquiert de la maison Price un vaste terrain. Pour subvenir aux frais de cet achat, Mgr de Québec est aidé par quelques prêtres et par une souscription populaire d'un sou par personne, dans tout le diocèse de Québec ${ }^{10}$.

Le 4 a aût 1875 restera une date mémorable dans les annales du Séminaire de Chicoutimi, car c'est la bénédiction solennelle du nouveau Séminaire et d'un magnifique tableau représentant la sainte Famille,

7 A.S.C., Ann., vol. I, 2, 3, 4, 5.

8 A.S.C., Ibid., 10-11.

9 Ibid., 8.

10 A.S.H.S., Mgr Eugène Lapointe, Le Séminaire de Chicoutimi 1873-1925, dans Almanach de l'Action Sociale Catholique, 1926, p. 47. 
donné par le Supérieur D. Racine ${ }^{11}$. A cette occasion, Mgr l'Archevêque de Québec fait l'éloge de son grand vicaire et reconnaît devoir à M. Racine l'idée d'un séminaire au Saguenay; nous lisons à ce sujet dans les annales : a Mgr fit ensuite l'éloge de son Grand Vicaire, qui venait de couronner tant d'œuvres de zèle et de dévouement dans le Saguenay par la construction du Séminaire ${ }^{12}$.

A partir du 8 novembre 1875, pour répondre au désir de son Archevêque, le Supérieur réside au Séminaire même, dans un appartement préparé au premier étage, dans l'extrémité nord-est de la bâtisse ${ }^{13}$.

Il était ainsi en mesure, écrit l'abbé Huard dans l'Apôtre $d u$ Saguenay, de suivre et de diriger l'œuvre qui n'était encore qu'à ses débuts. S'astreignant lui-même à toutes les exigences de la vie de communauté, il s'étudia constamment à établir et à fixer l'esprit et les traditions de la maison ${ }^{14}$.

Lors d'une réception, le 28 mars 1878, offerte au Supérieur du Séminaire, une adresse lui est lue en ces termes :

Eh ! bien-aimé Supérieur, n'êtes-vous pas comme notre père? $\mathrm{Ne}$ veillez-vous pas sur nous avec la même bonté et la même sollicitude qu'un père? Vous nous avez prodigué tous les jours toutes sortes de bienfaits ${ }^{15}$...

Le curé Racine ne néglige pas pour autant ses fonctions de curé et de vicaire général; la besogne à abattre est énorme mais il veille surtout sur son Séminaire et se montre soucieux d'en compléter l'organisation. Dans une lettre datée du 8 juin 1877, il apprend de Mgr T.E. Hamel que ses démarches sont exaucées et "que le Séminaire de Chicoutimi est affilié à l'Université Laval ${ }^{16}$ ».

C'est au milieu de toutes ces activités que le curé Dominique Racine apprend, le 15 mai 1878 - au moment de partir pour le concile provincial où il est invité comme théologien, - qu'il apprend, dis-je, son élection au siège épiscopal du nouveau diocèse de Chicoutimi ${ }^{17}$.

Revêtu de l'épiscopat, Mgr Dominique Racine voit son champ d'action subitement agrandi. Il lui faut, en plus des réalisations à peine esquissées, établir solidement le diocèse de Chicoutimi et le doter d'institutions nécessaires, en plus de fonder de nouvelles paroisses. Mais il a létoffe d'un pionnier, d'un bâtisseur et les dix années de son épiscopat seront fort bien remplies.

Le nouvel évêque demeurera supérieur du Séminaire jusqu'en 1882 et l'expansion, la consolidation de cette œuvre sera toujours au premier plan de ses activités.

11 A.S.C., Ann., vol. I, p. 23.

12 Ibid., p. 25.

13 Ibid., p. 35.

14 V. A. Huard. L'apôtre du Saguenay, p. 43.

15 A.S.C., Ann., vol. I, 147. 8 juin 1877.

16 A.S.Q., Université 41, no 38, lettre de Mgr T.E. Hamel à l'abbé D. Racine, 17 A.S.C., Ann., vol. I, 170-171. 
Dès 1879, par un acte du Parlement provincial, il fait ériger le Séminaire de Chicoutimi en corporation civile. ${ }^{18}$. Le 25 décembre 1880 , par un mandement, l'évêque de Chicoutimi établit la Congrégation de la Sainte-Vierge au Petit Séminaire ${ }^{19}$. Enfin, en 1881, il désigne saint Thomas d'Aquin comme patron des études.

Mais Mgr D. Racine est désormais évêque d'un grand diocèse, il ne peut l'oublier. Pour se consacrer davantage à toute sa tâche d'évêque, de pasteur et de père du petit' peuple saguenéen, il songe à se démettre

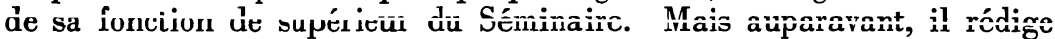
lui-même la Constitution concernant le gouvernement et l'administration de cette maison; elle entrera en vigueur le 21 janvier 1882 . Dans ces règlements introduits par Mgr Racine, québécois d'origine, par le programme des études, par les méthodes, par l'esprit et même par le costume, le Séminaire de Chicoutimi devient une sorte de réplique du Séminaire de Québec ${ }^{20}$. Il n'y a pas de doute possible, l'organisation et le développement du Séminaire requièrent de la part du premier supérieur beaucoup de temps et de soin. Il y met tout son cœur et toute son intelligence. Ayant abandonné ce poste, il se permet d'écrire, en décembre 1883, des notes fort à propos sur les devoirs des professeurs, des régents et des maîtres de discipline du Petit Séminaire de Chicoutimi.

\section{CONCLUSION}

Le 5 octobre 1887, Mgr Dominique Racine quitte définitivement le Séminaire pour habiter le premier et modeste évêché provisoire. Malgré les instances et les supplications de ses prêtres et l'extrême douleur que le fondateur ressent, il accepte la séparation pour laisser plus d'espace à la communauté grandissante. Le Séminaire de Chicoutimi doit beaucoup à son fondateur et premier supérieur, car ce dernier jette presque seul les bases d'une institution d'une importance fondamentale pour la région. Il semble que $\mathrm{Mgr}$ Taschereau s'en remet exclusivement à $\mathrm{M}$. le curé Racine, ne lui prêtant au début que quelques séminaristes bénévoles qu'il rappelle d'ailleurs un an ou deux plus tard. A l'organisateur de cette œuvre primordiale, à cet homme de premier plan manquera l'assistance de quelques prêtres choisis avec soin parmi les mieux doués et les plus aptes à collaborer à cette ouvre. Jusqu'à la fin de sa vie d'ailleurs, il éprouve une grande inquiétude car il réalise, plus de quinze ans après la fondation de son Séminaire, que cette œuvre n'est pas assez solidement assise. Il ne faut pas oublier que le Saguenay est encore à cette époque très isolé et que s'y enfoncer, même pour une cause aussi noble que celle de l'éducation, n'attire pas tous les hommes. La pauvreté du Séminaire de Chicoutimi est assez marquée, ce qui ne l'empêche pas de jouir d'un grand prestige auprès de la population ${ }^{21}$.

18 A.S.H.S., dossier 738, pièce 3, V.S., vol. I, 40.

19 A.S.C., Ann., vol. I, 461-473.

20 A.S.H.S., Document 813, p. 143.

21 A.S.H.S., document 813, p. 151, 155, 163, 189 
Ce séminaire est né du dévouement, des sacrifices du curé Racine. Ses quinze premières années d'existence se ressentent de toute la situation de la région, il grandit lentement à travers les difficultés nombreuses; mais ce que le premier évêque de Chicoutimi a fondé dans la pauvreté ne doit pas disparaître.

Le Séminaire de Chicoutimi continuera à grandir - toujours fidèle à la mission de son fondateur et père, il restera le " joyau » de l'œuvre éducationnelle de l'Eglise diocésaine.

Jean-Claude Drolet, professeur. Centre d'études et de recherches historiques du Saguenay, Chicoutimi. 